\title{
From Extraneous Noise to Categorizable Signatures: Using Multi-scale Analyses to Assess Implicit Interaction Needs of Older Adults with Visual Impairments
}

\author{
Kevin P. Moloney, V. Kathlene Leonard, Bin Shi, \\ Julie A. Jacko, Brani Vidakovic, and François Sainfort \\ School of Industrial \& Systems Engineering, Georgia Institute of Technology, \\ 765 Ferst Drive NW, Atlanta, GA, 30332-0205, USA \\ \{kmoloney, vkemery, bshi, jacko, brani, \\ sainfort\} @isye.gatech.edu
}

\begin{abstract}
The holistic understanding of human-computer interaction (HCI) is increasingly important, especially given the impending influx of older users who present dynamic needs that evolve with age. This study explores pupillary response behavior (PRB) during computer interaction to identify underlying differences between older adults of varying ocular profiles. PRB was measured from two groups of individuals diagnosed with Age-related Macular Degeneration (AMD) and a visually healthy control group. Unconventional analytical techniques - wavelet-based multifractal analyses - were used to identify PRB anomalies resulting from the effects of aging and/or ocular pathology. A distribution of regularity indices was extracted from the data signals to reveal signatures of PRB change patterns. One characteristic of the multifractal spectrum, Left Slope (LS), fully distinguished the user groups, revealing trends of increasing PRB irregularity with increasing levels of ocular dysfunction.
\end{abstract}

\section{Introduction}

The holistic characterization of users, including both implicit and explicit interactions, has potential to inform system design in order to better anticipate and account for user needs across HCI scenarios. This is critical considering the impending influx of older adult users who present a highly dynamic array of needs and capabilities that evolves with age. A recent study [1] revealed consistent performance differences between users with and without Age-related Macular Degeneration (AMD), despite the absence of significant user differences on traditional demographic and clinically-assessed measures of visual function (e.g., visual acuity). This suggests that aspects of users' visual health and capabilities can manifest themselves as impediments to HCI, while remaining undisclosed through traditional user profiling efforts.

One popular objective method to convey covert aspects of $\mathrm{HCI}$ is the interpretation of psychophysiological responses to stimuli, including examination of constriction and dilation of the iris (i.e., pupillometry) during task performance. Research has long supported that changes in pupil diameter correspond to information acquisition, processing and cognitive workload (e.g., larger pupil = higher levels of processing) [2]. 
More recently, research in HCI has used pupillary activity during computer-based interactions to predict the cognitive demands experienced by users during performance of varying tasks to help inform interface and interaction design [3].

However, the proper measurement and interpretation of pupillary response behavior (PRB) is not straightforward. PRB is affected by a variety of factors, including ambient light, fatigue, and medication use [2]. Moreover, aging naturally leads to ocular changes, such as shrinking pupil size and slower, smaller responses to visual stimuli [2]. Ophthalmologic research has also revealed that ocular disease (e.g., AMD) can decrease controlled, sustained movement of the iris [2]. Thus, powerful analytical tools are needed to detect these muted or irregular PRB change patterns.

The most commonly used statistical methods for analyzing PRB include: mean task-evoked pupillary response (TEPR); averaging measures of pupil diameter for a given post-stimulus time period; and frequency, power and spectral analyses. These approaches, however, do not adequately account for the seemingly erratic nature of PRB intrinsic to the pupil's physiological control mechanisms. Furthermore, they cannot resolve the amplified PRB complexity and irregularity exhibited by older adults with visual impairment. The richness of PRB in these individuals requires more sophisticated statistical techniques to improve the ability to examine this facet of HCI.

This study uses an advanced statistical concept - wavelet-based analysis of multifractality - to leverage the richness of this population's pupillary response data. The multifractal spectrum refers to the probability distribution of Hölder regularity indices ( $\alpha$ 's) from the raw data signal [4]. These regularity indices represent estimations of signal smoothness, based on fluctuations and correlations within local neighborhoods of time-series data points. Geometric attributes of multifractal spectra can be used to describe the richness of signal irregularity across scales of resolution. One such measure is Left Slope (LS), with a steeper slope indicative of decreasing multifractality and a smoother data signal with smaller, slower, or more persistent changes.

\section{Methods}

Pupillary response behavior was measured from older adults (mean age $=76$ yrs) diagnosed with AMD, the leading cause of visual impairment in the US and UK. As summarized in Table 1, participants were grouped according to ocular disease and visual acuity, including two user groups of individuals diagnosed with AMD and one visually healthy control group. Comprehensive ophthalmologic exams were provided to ensure the clinical diagnosis of AMD and knowledge of current visual capabilities. Tasks were performed under conditions of best-corrected vision. PRB was measured with the Applied Science Laboratories ${ }^{\circledR}$ Model $501^{\circledR}$ head-mounted optics system.

Table 1. User group characterization

\begin{tabular}{cccc}
\hline Group & $\mathrm{n}$ & Visual Acuity (Range) & AMD Diagnosis? \\
\hline Control & 14 & {$[20 / 20-20 / 32]$} & No \\
Group 1 & 8 & {$[20 / 32-20 / 70]$} & Yes \\
Group 2 & 6 & {$[20 / 80-20 / 200]$} & Yes \\
\hline
\end{tabular}


Participants performed 105 trials of a computer-based task; a single trial entailed the drag and drop of a Microsoft ${ }^{\circledR}$ Word file icon to a Window folder icon, integrated with different types of multimodal feedback to assist the participants' drop of file into the folder. Task-based PBR of each participant was recorded at a rate of $60 \mathrm{~Hz}$. This study employed a repeated-measures, within-subjects design, with each participant receiving all experimental conditions (e.g., feedback forms). User group was the independent, between-subjects variable. A more detailed methodological description and performance summaries can be found in related work [1].

The wavelet-based multifractal analysis approach is best summarized by a five-step procedure, including: 1) The preparation and segmentation of individual pupillary response behavior data signals; 2) The application of a discrete wavelet transform; 3) The generation and characterization of a multifractal spectrum for each data set; 4) The regression-based estimate of the LS measure; and 5) ANOVA and post-hoc analysis of group-based differences. As a comprehensive account is not permissible within this brief manuscript, interested readers are directed to related work $[4,5]$.

\section{Results and Conclusions}

Analysis revealed that decreases in LS correspond to decreases in the ocular condition (in terms of both disease and acuity) of users. Figure 1 illustrates this clear monotonic trend between the LS multifractal summary and ocular condition. One-way analyses of variance (ANOVA) confirmed significant group-based differences with respect to LS $(F=32.258, p<0.01)$. Post-hoc comparisons (Games-Howell) further revealed significant differences in LS between all user groups $(\mathrm{p}<0.05)$, in accordance with the monotonic trend. Group 2's PBR can be classified as the most multifractal, with a relatively rough PBR signal containing larger, faster, and anti-persistent changes in pupil diameter, followed by Group 1 and then the Control Group.

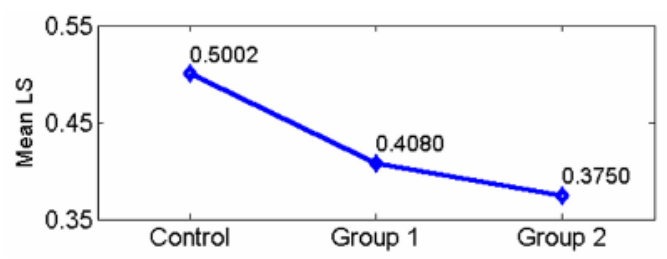

Fig. 1. Monotonic relationship between multifractality and ocular profile

To further reveal the contribution of this novel analytical approach, Figure 2 presents a comparison of individuals without and with AMD. Traditional statistical methods (e.g., the TEPR) would produce an interpretation that these individuals exhibit equivalent PRB and experience similar cognitive load during task performance (see Figures $2 \mathrm{a}$ and $2 \mathrm{~b}$ ). However, using multifractal analysis to examine the underlying signatures of irregularity, a clearer distinction can be made between these individuals (see Figures $2 \mathrm{c}$ and 2d). The individual with AMD (Figure 5d) exhibits PRB that is much more multifractal, based on LS and the resultant multifractal spectrum. 

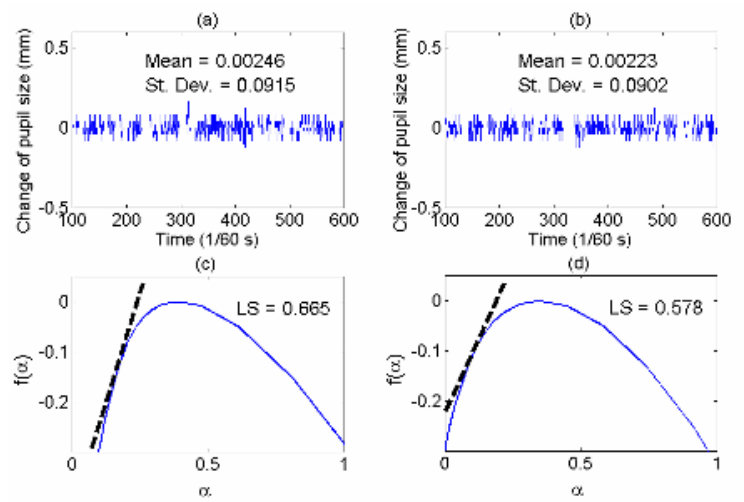

Fig. 2. Illustration of the utility of multifractal analysis. Figures (a) and (b) plot the PRB of individuals without and with AMD, respectively, during computer-based task performance. Figures (c) and (d) illustrate the corresponding multifractal spectra from these same datasets.

This research has potentially far-reaching implications for the examination of cognitive workload via PRB and furthering the understanding of the interaction needs and behaviors of users who are aging and/or have visual impairment. Multifractal analyses provide a new window through which the implicit subtleties of PRB can be utilized by HCI researchers. Additional efforts are underway to classify these individuals on the basis of other attributes of fractality. These novel analytical methods extend HCI user characterization beyond the use subject demographics or performance measures. The application of these analytical techniques holds great promise for utilizing the richly informative PRB of older adults with visual impairment in the context of HCI.

\section{Acknowledgments}

This research was made possible through funding from the National Science Foundation (BES-9896304), and the National Security Agency (NSA E24-60R).

\section{References}

1. Jacko, J.A., Moloney, K.P., Kongnakorn, T., Barnard, L., et al.: Multimodal feedback as a solution to ocular disease-based user performance decrements in the absence of functional visual loss. Int. J. of HCI 18(2) (2005) 183-218

2. Loewenfeld, I.E.: The Pupil: Anatomy, Physiology, and Clinical Applications. 2nd edn. Butterworth-Heinemann, Oxford, UK (1999)

3. Iqbal, S.T., Zheng, X.S., Bailey, B.P.: Task-evoked pupillary response to mental workload in human-computer interaction. In: Proceedings of CHI'04. ACM Press (2004) 1477-1480

4. Gonçalvès, P., Riedi, R., Baraniuk, R.: A simple statistical analysis of wavelet-based multifractal spectrum estimation. In: Proceedings of the 32nd Asilomar Conference on Signals, Systems and Computers. IEEE (1998)

5. Vidakovic, B.: Statistical Modeling by Wavelets. John Wiley \& Sons, New York, NY (1999) 